\title{
The emergence of antimicrobial resistance in enterococci isolates from infants: A review study \\ Asem A. Shehabi ${ }^{1}$, Emman F Badran², Nazha Ala'a Alnasra'
}

1 Department of Pathology-Microbiology, Faculty of Medicine, University of Jordan, Amman, Jordan.

\section{Abstract}

The emergence of antibiotic resistance in potential bacterial pathogens is considered as an important consequence of antibiotic misuse and overuse in humans and animals. In addition, lacks of adequate knowledge about proper using of antimicrobial drugs as well as infectioncontrol practice in hospital and medical care settings have led to the continuing development of extensive antimicrobial resistance problems worldwide. Certain multidrug-resistant commensal and potential pathogens are now widely spread in community and hospital patients. Examples are meticillin-resistant Staphylococcus aureus (MRSA), vancomycin-resistance enterococcci (VRE), extended-spectrum $\beta$-lactamase and carbapenemase-producing coliforms, floroquinolone-resistant and toxin-hyperproducing Clostridium difficile. During the last decade, Enterococcus faecalis and Enterococcus faecium were the most prevalent species cultured from human infections. Incidence of resistant to multiple antibiotics in these two species has become increasingly common in the hospitalized patients all over the world, particularly $E$. faecium is reported to be responsible for most vancomycin-resistant enterococci (VRE) infections.

This short review is focusing on the recent emergence of antimicrobial resistance among enterococci isolates from infants and the available option of their infection treatment.

Keyword: Enterococci resistance, VRE, Infants, Jordan 


\section{Introduction}

Enterococci are common communsals of human and animal intestinal microflora. Enterococci are also among the frequent early colonizers in the intestine of breastfed infants [1]. E.faecalis and E. faecium are the most prevalent species cultured from human infections and are increasing to become associated with nosocomial infections [2-3]. The emergence of vancomycin- resistance enterococci (VRE) during recent years has seriously affected the treatment and infection control of these organisms. VRE, particularly $E$ faecium strains, are frequently resistant to all antibiotics that are effective for the treatment of vancomycin-susceptible enterococci. This new development leaves clinicians treating VRE infections with limited therapeutic options [4-5].

An increase in the prevalence of E.faecium has been recently observed to occur among neonatal patients in ICUs of many countries. This shift is likely to be explained in part by the rapid emergence of VRE [3-4].

Enterococci are intrinsically resistance to many antibiotics, including the following agents: semisynthetic penicillins such as oxacillin, cephalosporins of all classes, monobactams and polymyxins. Aminoglycosides show insusceptibility at a low level, most probably due to a reduced uptake. In addition, enterococci are relatively resistant to chemical disinfectants such as chlorine, gluteraldehyde and alcohol [6-7].

Enterococci may develop increased resistance to penicillins through acquisition of $\beta$-lactamases or PBP/5 mutations [7 ]. High-level penicillin and ampicillin resistance in $E$. faecium is mediated via point mutations in the house-keeping pbp5 gene leading to reduced penicillin binding to the expressed protein [8]. Almost exclusively strains of $E$. faecalis can express $\beta$ - lactamase enzymes that confer high level resistance against all penicillins, except those combined to $\beta$-lactamase inhibitors (sulbactam or clavulanate). The enterococcal penicillinase gene is often found on a transferable plasmid [8] .

While intrinsic mechanisms result in low level aminoglycoside resistance, acquisition of mobile genetic elements typically contributes to high-level aminoglycoside resistance in both $E$. faecium and E. faecalis [9].Only the two aminoglycosides; gentamicin and streptomycin show a synergistic effect when given in combination with a cell-wall active agent like a penicillin or glycopeptides [9]. Certain aminoglycoside-modifying enzymes mediate highlevel gentamicin and streptomycin resistance in $E$. faecalis and E. faecium [9]. Glycopeptide antibiotics, vancomycin and teicoplanin, are used in the treatment of serious enterococci infections in cases of resistance or allergy to $\beta$-lactams. Resistant strains which are responsible for colonization or infection have been isolated with an increasing frequency from patients in the presence or absence of glycopeptide therapy [10-11]. Vancomycin and teicoplanin inhibit cell wall synthesis through binding to the Dalanyl-D-alanine ( $D$-ala-D-ala) terminus of the peptidoglycan precursors which prevents this precursor from participating further in the synthesis of cell wall [11]. According to the Clinical and Laboratory Standards Institute (CLSI), the following are the MIC interpretative criteria for vancomycin in enterococci: (i)susceptible $\leq 4 \mu \mathrm{g} / \mathrm{ml}$, (ii)intermediate-8-16 $\mu \mathrm{g} /$ $\mathrm{ml}$,(iii)resistant $\geq 32 \mu \mathrm{g} / \mathrm{ml}$ [12].

Enterococci carry a wide variety of mobile genetic elements, and are considered to be a reservoir for the antibiotic resistance genes acquired by other gram-positive bacteria [13]. Both E.faecalis and E. faecium either isolated from human or dairy products at the same time showed fast similar resistance patterns against a wide variety of antibiotics, including glycopeptide such as vancomycin and teicoplanin [14]. Widespread use of vancomycin and extended spectrum cephalosporins in hospitals is likely to have contributed to the emergence and dramatic increase of VRE over the past 20 years [15]. VRE may 
be transmitted from patient to patient by the hands of healthcare workers and medical devices and often have virulence traits that enhance their ability to colonize rapidly hospitalized patients leading to the development of serious infections [14-16]. Different types of vancomycin-resistance genes have been reported in enterococci. Glycopeptide-resistant genotypes in enterococci include VanA (high-level resistance), VanB and VanD with moderate to high-level resistance and VanC (C1, C2, C3) causing intrinsic low-level resistance [2].

In Europe and United States, the 3 most common vancomycin-resistance enterococci phenotypes detected were VanA, VanB, and VanD. Enterococci VanA isolates exhibit high-level resistance to both vancomycin and teicoplanin, while VanB isolates have variable resistance to vancomycin and remain susceptible to teicoplanin. The VanC phenotype is mediated by the chromosomal VANC1 and VANC2 genes, which are constitutively present in E. gallinarum (VANC1) and $E$. casseliflavus (VANC2). These genes confer relatively low resistance levels to vancomycin and are not transferable. To date, the VanD, VanE, and VanG phenotypes have been described in only a few strains of enterococci [17].

\section{Epidemiology of enterococci resistance in infants}

A recent study in Pakistan reported by Yameen et al. [18], evaluated the incidence of nasal and rectal colonization of vancomycin-sensitive and resistant enterococci in patients of pediatrics ICU (PICU) The nasal and rectal swab samples were collected from 110 patients admitted in PICUs in three tertiary care hospitals of Rawalpindi Medical College, Pakistan. The identification of enterococci was done by biochemical tests and by PCR for $d d l$, vanA and vanB genes. Antibiotic susceptibility testing was performed by disc diffusion and MICs were determined for vancomycin, tetracycline, ciprofloxacin and oxacillin. Out of 220 nasal and rectal samples, 9 vancomycin-resistant enterococci (VRE) and 76 vancomycin-susceptible enterococci (VSE), consisting of 40 E. faecalis and 45 E. faecium. Generally, E. faecium isolates were more resistant than $E$. faecalis. MICs of vancomycin for nasal and rectal VRE were $512 \mathrm{mg} / \mathrm{L}$ and 64 to $512 \mathrm{mg} / \mathrm{L}$, respectively.

A study from Mexico by Ochoa, et al. [19], has investigated distribution of multidrug-resistant Enterococcus faecium isolates in a tertiary care center. Twelve clinical vancomycin-resistant Enterococcus faecium (VREF) isolates were obtained from pediatric patients at the Hospital Infantil de México Federico Gómez (HIMFG). Among these VREF isolates, $58.3 \%$ (7/12) were recovered from urine, while $41.7 \%$ (5/12) were recovered from the bloodstream. The VREF isolates showed a $100 \%$ rate of resistance to ampicillin, amoxicillin-clavulanate, ciprofloxacin, clindamycin, chloramphenicol, streptomycin, gentamicin, rifampicin, erythromycin and teicoplanin. In addition, $16.7 \%(2 / 12)$ of the isolates were resistant to linezolid, and $66.7 \%(8 / 12)$ were resistant to tetracycline and doxycycline. PCR analysis revealed the presence of the vanA gene in all 12 VREF isolates. A study from Greece demonstrated high incidence of antibiotic resistance in enterococci isolated from neonates' gut microbiota [20]. The study showed the prevalence of fecal enterococci in Greek healthy infants and their susceptibility to antimicrobial agents as well as the presence of genetic determinants encoding for certain antibiotic resistance traits. A total of 263 fecal samples derived from 97 infants were collected on day 4, 30, and 90 after delivery. E. faecalis was the most frequently identified species (54.6\%) followed by E. faecium, while E. casseliflavus, E. flavescens and E. gallinarum were also traced. The isolates were examined for their resistance to 12 antibiotics. Rifampicin resistance was the highest observed (53.2\%), followed by resistance to tetracycline $(42.0 \%)$, erythromycin (35.7\%), and vancomycin (11.2\%). Multiresistant strains were highly prevalent. Only intrinsic vanco- 
mycin resistance (vanC1 and vanC2/C3 genes) was traced. The ermB gene was detected in 49 out of 96 erythromycin-resistant isolates, while tet genes were detected in 51 out of 113 tetracycline-resistant strains, with tet $(\mathrm{L})$ being the most frequently observed. The study conclude that antibiotic-resistant enterococci are already established in the fecal microbiota of healthy neonates, from the first days of an infant's life.

The study of Werner and his colleagues in Germany [4], has described an ongoing high prevalence of VanB type E. faecium in a neonatal ICU .During a 10 months' key period $71 \mathrm{E}$. faecium isolates including 67 vanB-type isolates from 61 patients were collected non-selectively. Vancomycin resistance was determined by different MIC methods (broth microdilution, VitekW 2) including two Etest protocols (McFarland 0.5/2.0. on Mueller-Hinton/Brain Heart Infusion agars). Performance of three chromogenic VRE agars to identify the vanB type outbreak VRE was evaluated. Majority of vanB isolates ( $n=56$, 79\%) belonged to a single ST192 outbreak strain type.Vancomycin MICs in cation-adjusted $\mathrm{MH}$ broth revealed a susceptible value of $\leq 4 \mathrm{mg} / \mathrm{L}$ for 31 (55\%) of the 56 outbreak VRE isolates. Etest vancomycin on $\mathrm{MH}$ and $\mathrm{BHI}$ agars revealed only two vanB VRE isolates with a susceptible result.

A second study from Germany by Hufnagel, et al. [21], has analyzed enterococcal colonization and antimicrobial susceptibility among infants in a neonatal intensive care unit. Over a 12-month period, they performed a prospective epidemiological study in 274 infants admitted to a neonatal intensive care unit. Twenty-three percent of the infants were colonized with enterococci. The three most common enterococcal species were $E$. faecium (48\% of isolates), E. casseliflavus (25\%) and E. faecalis (13\%). Fifty-seven percent of the enterococci found were resistant to three of five antibiotic classes, but no vancomycin-resistant isolates were observed. In 63 cases $(23 \%)$, enterococci were isolated from either body cultures or from the first meconium. Differen- tiation at the species level showed 30 (48\% of all enterococcal isolates) E. faecium, $16(31 \%)$ E. casseliflavus, eight (12\%) E. faecalis, two (3\%) E. durans, and seven (11\%) undifferentiated enterococcal species. Thirty-six isolates (57\% ) of all enterococcal isolates were resistant to three of the five antibiotic classes tested (aminopenicillins, carbapenems, aminoglycosides, sulfonamides/folinic acid antagonists and vancomycin) and were therefore classified as multidrug-resistant. All of these multidrug-resistant isolates were resistant to aminopenicillins and aminoglycosides. Of the 36 multidrug-resistant enterococci, 35 isolates were resistant to both sulfonamides and carbapenems. In addition to the 36 multidrugresistant isolates, five other isolates were resistant to aminoglycoside, and all of them displayed low-level aminoglycoside resistance. None of the enterococci was vancomycin- resistant. Twenty-four (80\%) of $E$. faecium, 11 (69\%) of E. casseliflavus, and one (13\%) $E$. faecalis isolate were multidrug-resistant.

The study of Dahlen et al. in Sweden [22], evaluated the presence of virulence factors and antibiotic susceptibility among enterococcal isolates from oral mucosal and deep infections. Forty-three enterococcal strains from oral mucosal lesions and 18 from deep infections were isolated from 830 samples that were sent during 2 years to Oral Microbiology, University of Gothenburg, for analysis. MIC determination was performed using the E-test method against the most commonly used antibiotics in dentistry, for example, penicillin $\mathrm{V}$, amoxicillin and clindamycin.Vancomycin was included in order to detect vancomycin-resistant enterococci (VRE) strains.

Sixty strains were identified as Enterococcus faecalis and one as Enterococcus faecium. Ampicillin and amoxicillin showed the strongest effect on the enterococci but only 57.4 and $31.1 \%$, respectively, of the isolates were susceptible, as screened routinely by the disc diffusion method. Using the E-test method for estimation of MIC values, all of the E. faecalis strains were susceptible as well as the $E$. faecium 
isolate for amoxicillin. Of the strains from mucosal and deep infection samples, 90\% showed minimal inhibitory concentrations $\left(\mathrm{MIC}_{90}\right)$ of $256 \mathrm{mg} / \mathrm{ml}$ or more against clindamycin.all enterococcal strains were sensitive to vancomycin.

The study of Solheim, et al. in Norway [23], has showed the wide distribution of virulence genes and antibiotic resistance profiles of community-derived E. faecalis isolated from the feces of Norwegian infants. Tetracycline resistance was the most prevalent resistance trait among the baby isolates (17/31). Moreover, a few of the isolates $(n=4)$ showed high level erythromycin and gentamicin resistance.

\section{General epidemiology of enterococci resistance in Arab countries}

According to our best knowledge by recent search of PubMed and other international indexing sources, we have not found a single study from any Arab country which has reported on enterococci infection or colonization in infants, while there are many studies reported about the general incidence and antimicrobial susceptibility of enterococci isolates from clinical specimens or nosocomial infections during the last decade (2003-2014). Most of these studies showed low to high incidence rates of resistance among E. faecalis and E. faecium clinical isolates including vancomycin- resistance (23-32). However, we have finished recently a study on antimicrobial resistance and virulence factors among $E$. faecalis and $E$. faecium colonizing intestines of Jordanian infants. This study will be published within the next few months.

Recommended treatment of enterococci infections The optimal treatment of enterococci bacteremia or VRE is ampicillin if the isolates is susceptible to this drug, whereas the best treatment in case of ampicillin resistance is still under investigation. Linezolid was successfully used in treatment of invasive VRE bacteremia, it has good activity against both $E$. faecalis and E. faecium, develop less resistance than other agents and can be given orally and or intravenously [33-34]. Linezolid, the first oxazolidinone, has a broad spectrum of activity against Gram-positive bacteria, including resistant strains such as MRSA and VRE. It has been approved by the USA Food and Drug Administration in 2002. The available literature data suggest that linezolid is a safe and effective agent for the treatment of serious Gram-positive bacterial infections in neonates and children [34].

\section{Competing interests}

The authors declare that they have no competing interests. 


\section{References}

1. K Orrhage, CE Nord. Factors controlling the bacterial colonization of the intestine in breastfed infants. Acta Paediatr Suppl 1999; 88: 47-57.

2. Miller WR, JM Munita, CA Arias. Mechanisms of antibiotic resistance in enterococci. Expert Rev Anti Infect Ther. 2014;12(10):1221-36.

3. Furtado I, Xavier PCN, LVM Tavares, F Alves, SF Martins, et al. Enterococcus faecium and Enterococcus faecalis in blood of newborns with suspected nosocomial infection, J Instit Trop Med Sao Paulo 2014; 56(1): 77-80.

4. Werner G, I Klare, C Fleige, U Geringer, W Witte, et al. Vancomycin-resistant vanB-type Enterococcus faecium isolates expressing varying levels of vancomycin resistance and being highly prevalent among neonatal patients in a single ICU. Antimicrob Resist Infect Cont 2012; 1: 21.

5. Arias CA, BE Murray. The rise of the Enterococcus: beyond vancomycin resistance. Nat Rev Microbiol 2012; 16:266-78.

6. Van Schaik W, RJ Willems. Genome-based insights into the evolution of enterococci. Clin Microbiol Infect 2010; 16(6):52732.

7. Hollenbeck $B L$, LB Rice. Intrinsic and acquired resistance mechanisms in enterococcus. Virulence 2012; 3: 421-569.

8. Rice LB. Antimicrobial resistance in gram-positive bacteria, American J Infect Cont. 2012; 34: S11-S19.

9. Chow J. Aminoglycoside resistance in enterococci, Clin Infect Dis 2000; 31:586 -9.

10. Wood AJ. Vancomycin resistant enterococcal infections. N Engl J Med 2000; 342:710-720.

11. Chavers LS. Vancomycin resistant enterococci: 15years and counting. J Hosp Infect 2003;53:159-171.

12. CLSI (2012), Performance Standards for Antimicrobial Susceptibility Testing, Twentieth InformationalSupplement, Wayne, PA: Clinical and Laboratory Standards Institute. CLSI document M100-S20.

13. Shankar N, AS Baghdayan, MS Gilmore. Modulation of virulence within a pathogenicity island in vancomycin resistant Enterococcus faecalis. Nature 2002; 417:746-750.

14. Cariolato D, C Andrighetto, A Lombardi. Occurrence of virulence factors and antibiotic resistances in Enterococcus faecalis and Enterococcus faecium collected from dairy and human samples in North Italy. Food Control 2008; 19: 886-892.
15. Tacconelli E, MA Cataldo. Review of vancomycin-resistant enterococci (VRE): transmission and control. Int J Antimicrob Agents 2008; 31:99-106.

16. Fisher K, Phillips $C$. The ecology, epidemiology and virulence of Enterococcus. Microbiology 2009; 155: 1749-57.

17. Deshpande LM, TR Fritsche TR, GJ Moet, et al. Antimicrobial resistance and molecular epidemiology of vancomycin-resistant enterococci from North America and Europe: a report from the SENTRY antimicrobial surveillance program. Diagn Microbiol Infect Dis 2007;58(2):163-70.

18. Yameen MA. S Iram, S Abdul Mannan, S Ali Khan, N Akhtar. Nasal and perirectal colonization of vancomycin sensitive and resistant enterococci in patients of paediatrics ICU (PICU) of tertiary health care facilities, BioMed Cent Infect Dis 2013;13:156.

19. Ochoa SA, G Escalona, A Cruz-Córdova, LB Dávila, Z Saldaña, et al. Molecular analysis and distribution of multidrug-resistant Enterococcus faecium isolates belonging to clonal complex 17 in a tertiary care center in Mexico City, BMC Microbiol 2013; 11:13291.

20. Kirtzalidou El,EK Mitsou, P Pramateftaki, A Kyriacou. Screening Fecal Enterococci from Greek Healthy Infants for Susceptibility to Antimicrobial Agents. Microb Drug Resist 2012; 18(6): 578585.

21. Hufnagel M, C Liese, C Loescher, M Kunze, H Proempeler, et al. Enterococcal colonization of infants in a neonatal intensive care unit: associated predictors, risk factors and seasonal patterns, BioMed Cent Infect Dis 2007; 7:107.

22. Dahlen G, S Blomqvist, A Almstahl, A Carlen. Virulence factors and antibiotic susceptibility in enterococci isolated from oral mucosal and deep infections. J Microbiol 2012; 4:10.

23. Solheim M, A Aakra, LG Snipen, I Nes. Comparative genomics of Enterococcus faecalis from healthy Norwegian infants, BioMed Cent Genom 2009; 10: 194-205.

24. Hashem YA, AS Yassin, MA Amin. Molecular characterization of Enterococcus spp. clinical isolates from Cairo, Egypt. Indian J Med Microbiol. 2015; 33 Suppl:S80-6.

25. Shorman M, JA Al-Tawfiq. Risk Factors Associated with Vancomycin-Resistant Enterococcus in Intensive Care Unit Settings in Saudi Arabia. Interdiscip Perspect Infect Dis 2013, ID 369674, 4 pages.

26. Araj GF, AZ Avedissian, NS Ayyash, AH Bey, GR El Asmar, et al. A reflection on bacterial resistance to antimicrobial agents at a major tertiary care center in Lebanon over a decade. J Med Liban 2012; 60 (3): 125-35. 
27. Salem-Bekhit MM. IM Moussa, MM Muharram, FK Alanazy, $\mathrm{MH}$ Hefni. Prevalence and antimicrobial resistance pattern of multidrug-resistant enterococci isolated from clinical specimens, Indian J Med Microbiol 2012; 30:44-51.

28. Hijazi N. AA Elmanama, A Al-Hindi. Vancomycin-resistant enterococci in fecal samples from hospitalized patients and non-hospitalized individuals in Gaza City. J Public Health 2009; 17:243-249

29. Mahafzah A, I Abu-Khader, F Bakri. Characterization of Enterococci Causing Nosocomial Infections at the Jordan University Hospital Over a Five-year Period. Jordan Med J 2008;42 (4): 229-237.

30. Salah R. N Dar-Odeh, O Abu Hammad, AA Shehabi. Prevalence of putative virulence factors and antimicrobial susceptibility of E. faecalis isolates from patients with dental Diseases, BioMed Cent Oral Health 2008; 8:17.

31. Klibi N, K Ben Slama, Y Sáenz, A Masmoudi, S Zanetti, et al. Detection of virulence factors in high-level gentamicin-resistant Enterococcus faecalis and Enterococcus faecium isolates from a Tunisian hospital. Can J Microbiol 2007; 53(3):372-9.

32. Udo, E.E. N Al-Sweih, AO Phillips, TD Chugh. Species prevalence and antibacterial resistance of enterococci isolated in Kuwait hospitals, J Med Microbiol 2003; 52:163-8

33. Pranita DT, AJ. Hsu. Optimizing Therapy for Vancomycinresistant Enterococcal Bacteremia in Children. Curr Opin Infect Dis 2014; 27(6):517-527.

34. Garazzino S, PA Tovo. Clinical experience with linezolid in infants and children. J Antimicrob Chemother 2011;66 Suppl 4:iv23-iv41

\section{Comment on this article:}

\section{$f(3)$ in $8+S$. P}

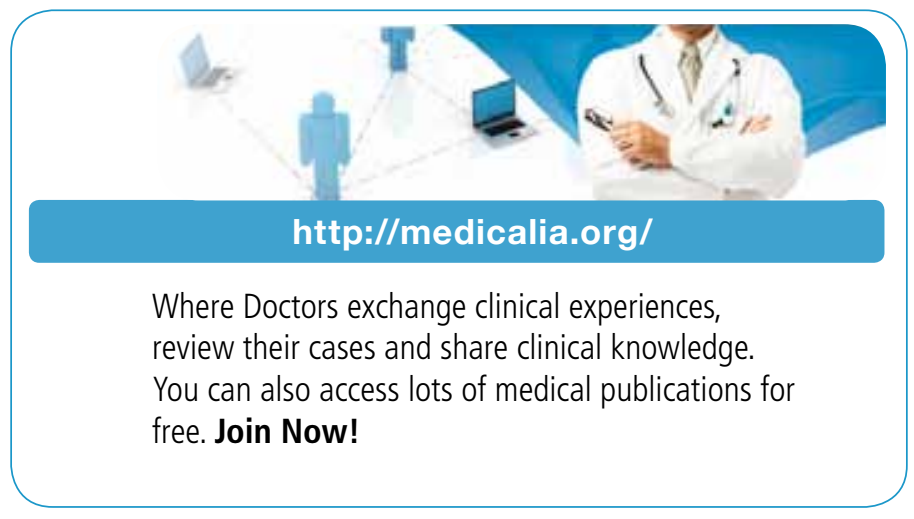

\section{Publish with iMedPub}

http://www.imed.pub

The Journal is an open access peer-reviewed journal that publishes scientific papers about all aspects of antimicrobials. The journal will publish original research articles, reviews, brief reports and case reports dealing with basic and clinical antibacterial agents, antiviral, antiprotozoals, antituberculuous, antifungal and antihelminthes agents.

All manuscripts must be prepared in English, and are subject to a rigorous and fair peer-review process. Accepted papers will immediately appear online.

The journal aims to advance the knowledge, attitude and the research of chemotherapy in the Arabic world in cooperation with international, national scientific and public societies as well as research centers with similar aims and objectives.

\section{Submit your manuscript here:} www.iajaa.org 